\title{
General immune dampening is associated with disturbed metabolism at diagnosis of type 1 diabetes
}

\author{
Anna Rydén ${ }^{1-3}$, Johnny Ludvigsson ${ }^{1}$, Mats Fredrikson ${ }^{4}$ and Maria Faresjö5,6
}

BACKGROUND: Type 1 diabetes (T1D) is a serious diagnosis with the prospect of grave short- and long-term complications and even death if poorly managed. An attempt has been made to describe how clinical and immunological deviations might influence each other close to the diagnosis of T1D.

METHODS: Sixty-nine newly diagnosed T1D children were studied together with a reference group of 30 healthy children. Cytokines (interleukin (IL)-6, IL-10, IL-13, IL-17, interferon- $\gamma$, and tumor necrosis factor- $\alpha$ ) were detected in in vitro culture by multiplex fluorochrome technique. Information of clinical status of the patients such as BMI, weight loss, pubertal stage, duration of symptoms, previous and/or ongoing infections, insulin requirement, and ketoacidosis were gathered together with the analysis of C-peptide and glycosylated hemoglobin (HbA1c).

RESULTS: In general, low cytokine secretion was found at diagnosis of T1D. However, high C-peptide, short duration of symptoms, or an infection prior to diagnosis was associated with increased immune activity including proinflammatory, Th2-associated, and Tr1-associated cytokines. In contrast, ketoacidosis and later pubertal stage at onset of disease were more related to a Th1-prone response.

CONCLUSION: There is a general immune dampening at diagnosis of T1D, which appears to be related to the metabolic state close to diagnosis.

$\mathbf{T}$ he $\beta$-cell destruction connected to type 1 diabetes (T1D) is usually rapid in young patients and might be more prolonged in adults developing the disease, but the rate may vary to a great extent between these two age groups (1). With a better $\beta$-cell function and more C-peptide, the less is the risk of ketoacidosis (2). A higher C-peptide at baseline and sustained levels at least a year after diagnosis have also shown to be beneficial to reduce the incidences of microvascular complications in T1D and to allow for intensive insulin treatment (to reach $\mathrm{HbAlc}<$ 7.5\%) together with fewer incidences of severe hypoglycemia (3). Furthermore, there are findings suggesting that C-peptide alters interactions between leukocytes and endothelium by causing a decreased upregulation of cell adhesion molecules and thereby reducing the rate of cell adhesion and migration (4), results that might indicate an inhibitory role of C-peptide in inflammation.
In the majority of T1D cases, autoantibodies toward one or more pancreatic islet proteins, such as insulin, glutamic acid decarboxylase $\left(\mathrm{GAD}_{65}\right)$, tyrosine phosphatases; IA-2 and IA-2 $\beta$; zinc transporter 8 (ZnT8) or the peptide antigen, corresponding to a conserved region of the heat-shock protein 60 (amino acids (AA) 437-460) are present, indicating the suggested autoimmune process (5-7).

Alterations in levels and patterns of various cytokines and chemokines have been associated with autoimmune diseases both in respect to beneficial and aggravating effects. T1D has been connected to a fluctuation in the T-helper (Th)1/Th2 balance, with an overproduction of Th1-associated cytokines that have been suggested to be prodiabetic and enhance the autoimmune process (8). Th1-like subtypes, secreting dominantly interferon (IFN)- $\gamma$ and tumor necrosis factor (TNF)- $\beta$, have been shown to be important for the destruction of the insulin-producing $\beta$ cells $(9,10)$. Proinflammatory cytokines, especially TNF- $\alpha$ and interleukin (IL)-6, have shown proof to home to the inflammatory site (11). Also, IL-17 immunity has been suggested in human T1D due to the findings of increased secretion and expression of IL-17, as well as an increased proportion of IL-17-secreting cells (12). In contrast, Th2-like cytokines, e.g., IL-4, IL-5, and IL-13 have been shown to be downregulated during this organ-specific autoimmune process (13-15). In addition, IL-10 secreted from regulatory $\mathrm{T}$ cells $(\operatorname{Tr} 1)$, and transforming growth factor- $\beta$ secreted by Th3 cells, inhibits Th1 cytokine synthesis (16).

Young children, and especially those diagnosed soon after infections, tend to have less remaining C-peptide (17). Besides this fact, little is known about the heterogeneity of T1D with regard to disease process and clinical course. With the gathered clinical and immunological information, an attempt has been made to describe how clinical features might be related to, and to influence, the outcome of T1D diagnosis.

\section{RESULTS \\ Low Cytokine Secretion at Diagnosis of T1D}

T1D children around the time of diagnosis had lower spontaneous secretions of all of the studied cytokines (IL-6, IL-10, IL-13, IL-17, IFN- $\gamma$, and TNF- $\alpha$ ) in comparison to

\footnotetext{
'Division of Paediatrics and Diabetes Research Centre, Department of Molecular and Clinical Medicine, Faculty of Health Sciences, Linköping University, Linköping, Sweden; ${ }^{2}$ Type 1 Diabetes R\&D Center, Novo Nordisk, Seattle, Washington; ${ }^{3}$ Pacific Northwest Diabetes Research Institute, Seattle, Washington; ${ }^{4}$ Linköping Academic Research Center, Faculty of Health Sciences, Linköping University, Linköping, Sweden; ${ }^{5}$ Biomedical Platform, Department of Natural Science and Biomedicine, School of Health Sciences, Jönköping University, Jönköping, Sweden; ${ }^{6}$ Division of Medical Diagnostics, Laboratory Medicine, Ryhov County Hospital, Jönköping, Sweden. Correspondence: Maria Faresjö (maria.faresjo@hhj.hj.se) 


\section{Articles | Rydén et al.}

the healthy children (Figure 1a-f). In vitro culture of a few, freshly handled, samples of peripheral blood mononuclear cells (PBMCs) collected from a small subgroup showed confirming results of a lower spontaneous secretion of IL-6,
IL-13, and TNF- $\alpha$ in T1D children compared to healthy children (Figure 2a-f).

In contrast, phytohemagglutinin (PHA)-induced response was found in all study subjects and for all the cytokines studied.
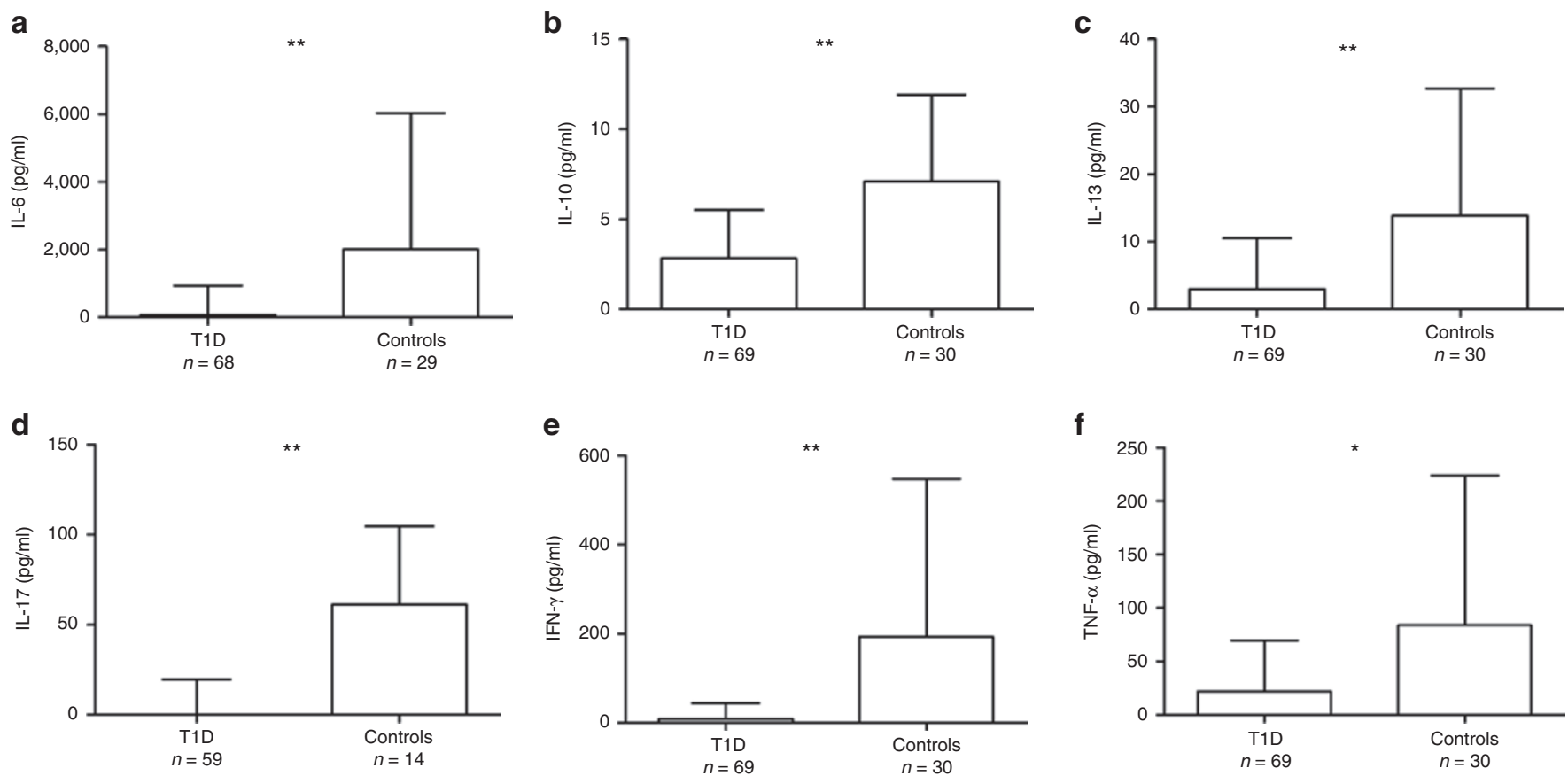

Figure 1. Spontaneous secretion of cytokines in newly diagnosed type 1 children and healthy children. Spontaneously secreted cytokines such as (a) interleukin (IL)-6 ( $\left.{ }^{* *} P<0.0001\right)$, (b) IL-10 (**P $\left.=0.004\right)$, (c) IL-13 $\left.{ }^{* *} P<0.0001\right)$, (d) IL-17 ( $\left.{ }^{* *} P=0.003\right)$, (e) interferon (IFN)- $\gamma\left({ }^{* *} P<0.0001\right)$, and (f) tumor necrosis factor (TNF)- $\alpha\left({ }^{*} P=0.01\right)$ were lower in children with newly diagnosed type 1 diabetes (T1D) in comparison to secretions seen from healthy children. Box plots are presented with median and interquartile range.

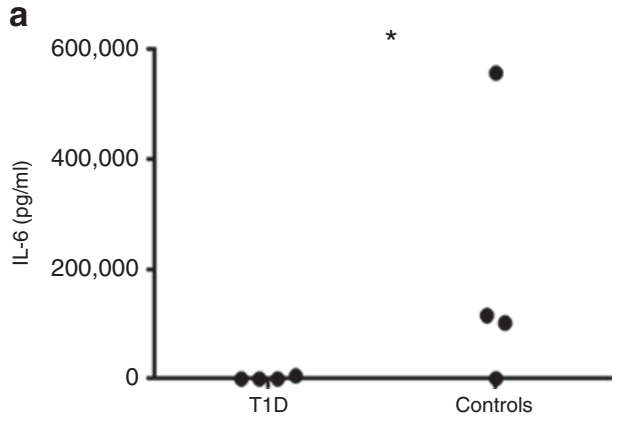

d

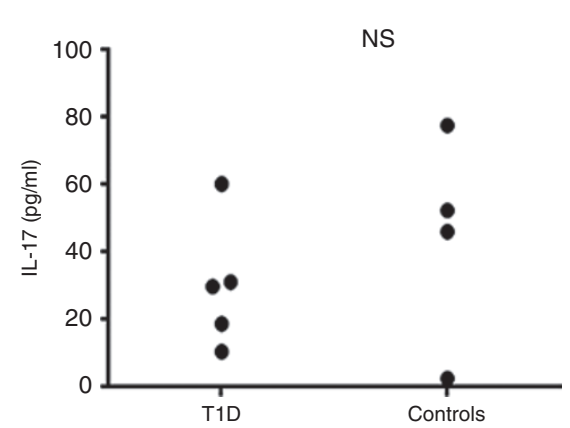

b

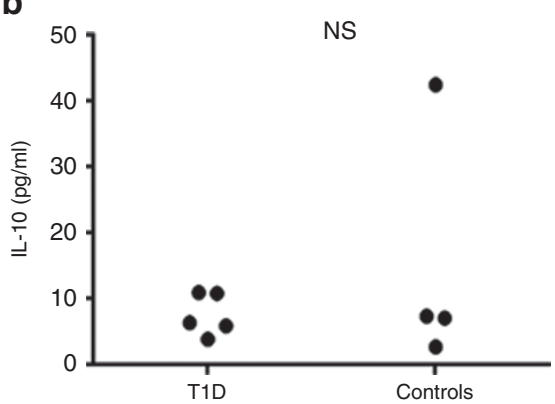

e

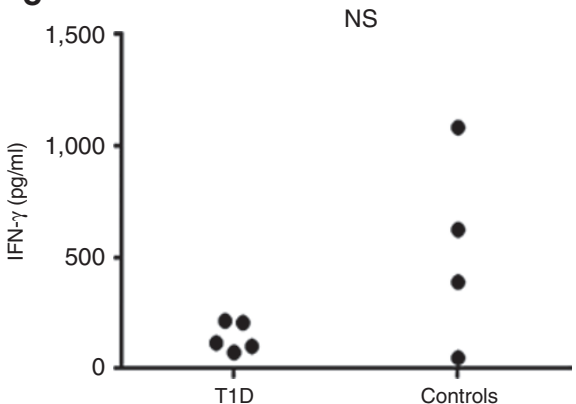

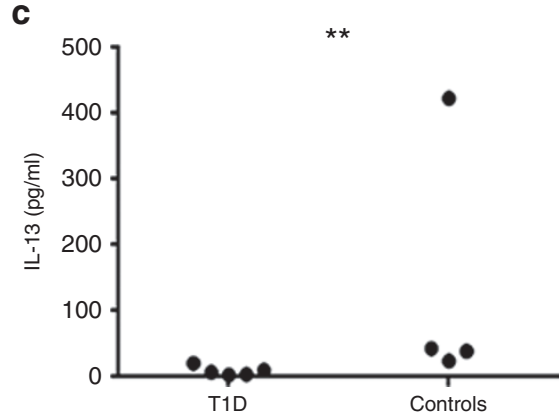

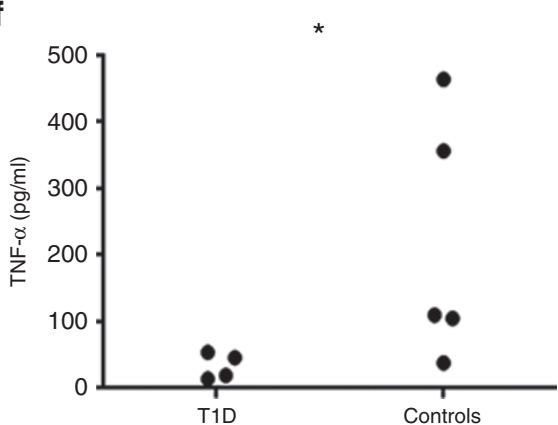

Figure 2. Spontaneous secretion of cytokines in newly diagnosed type 1 children and healthy children in a subsample of freshly handled peripheral blood mononuclear cells. Spontaneously secreted cytokines such as (a) interleukin (IL)-6 $\left(^{*} P<0.05\right)$, (b) IL-10 (not significant (NS)), (c) IL-13 (**P=0.01), (d) IL-17 (NS), (e) interferon (IFN)- $\gamma(\mathrm{NS})$, and (f) tumor necrosis factor (TNF)- $\alpha(* P=0.05)$ were lower in children with newly diagnosed type 1 diabetes (T1D) in comparison to secretions seen from healthy children. 
However, mitogen-induced secretion of IL-10 and IL-17 were generally lower in T1D patients $(P=0.005$ and $P=0.014)$, as were $\mathrm{GAD}_{65}$-specific secretions of IL- 6 and IFN- $\gamma(P=0.032$ and $P=0.013)$. Insulin peptide-specific secretion of IFN- $\gamma$, however, were higher from T1D patients compared to healthy controls $(P=0.013)$.

\section{C-peptide in Relation to Cytokine Secretion}

A higher C-peptide at diagnosis was associated with higher IL-13 secretions $(P=0.019$; Table 1$)$, which also tended to be true for spontaneous IL-10 secretions $(P=0.069$; Table 1$)$. Higher C-peptide values at T1D diagnosis were also related to higher spontaneous TNF- $\alpha$ secretion $(P=0.038)$, while lower TNF- $\alpha$ was connected to higher dosage of insulin per kilogram body weight, during the first $24 \mathrm{~h}$ following diagnosis $(P=0.005)$.

\section{Duration of Symptoms in Relation to Proinflammatory Autoantigen Response}

Higher $\mathrm{GAD}_{65}$ protein-specific TNF- $\alpha$ secretions were seen in children with a short duration of symptoms as compared to those with symptoms duration of 6 -wk and longer $(P=$ 0.006; Table 1). In the same line, IA-2-specific secretions of TNF- $\alpha$ were higher in those individuals whose diagnosis was not preceded by more than $2 \mathrm{wk}$ of symptoms duration $(P=0.021)$. Moreover, patients with symptoms duration of about 4 wk had higher PHA-induced TNF- $\alpha$ secretions $(P=$ 0.006). Spontaneously secreted TNF- $\alpha$ was higher in T1D children with a duration of symptoms longer than $6 \mathrm{wk}$ as compared to the individuals those had a shorter duration of symptoms before diagnosis $(P=0.028)$.

In contrast, $\mathrm{T} 1 \mathrm{D}$ diagnosis preceded by symptom durations of $12-16 \mathrm{wk}$ was connected to higher spontaneous IL-10 ( $P$ $<0.001$; Table 1$)$ and IL-13 secretions $(P<0.001$; Table 1$)$. However, observations observed in relation to a longer duration of symptoms were scarce in this material, why this connection should be regarded with some care. Mitogen-induced IL-13 secretion was higher in those individuals whose T1D diagnosis was preceded by $2(P=0.001)$ or $4(P=0.000)$ wk of symptoms duration and was higher in those with longer duration, even though this is uncertain due to the low number of observations.

\section{Ongoing Infection at Diagnosis in Relation to Cytokine and C-peptide Secretion}

Children and adolescents with an ongoing infection at the time of diagnosis had higher spontaneous secretions of IL-13 $(P<0.05)$ and a tendency to higher IL-10 secretions (Table 1). Autoantigen-stimulated IL-10 and IL-13 secretions were not influenced by the studied parameters (data not shown). In cases with an infection of $2 \mathrm{wk}$ before the diagnosis, higher TNF- $\alpha(P<0.001)$, and also IL-10 secretion $(P=0.009)$, were detected, but the longer the period prior to diagnosis that the patient had suffered an infection, the lower the measured spontaneous TNF- $\alpha$ secretion. The shorter the interval between preceding infection and T1D diagnosis, the lower the C-peptide levels $(P=0.036)$.

\section{Ketoacidosis and Pubertal Stage at Onset of Disease in Relation to Cytokine Secretion}

Individuals with ketoacidosis when diagnosed had a higher ratio of spontaneously secreted IL-10/IFN- $\gamma$ compared to those without ketoacidosis $(P=0.030)$, a difference that was further strengthened when withdrawing BMI as a contributing factor $(P=0.017$; Table 1$)$.

Children/adolescents diagnosed with T1D during pubertal stage IV had a higher ratio of IL-13/IFN- $\gamma$ compared to children diagnosed during pubertal stage I $(P=0.018)$. Patients diagnosed during the later stages of puberty (IV and V) responded with higher IFN- $\gamma(P=0.004)$ and TNF- $\alpha(P=$ 0.002) secretions to PHA stimulation than those diagnosed during the early- or prepubertal stages.

\section{Correction for Multiple Comparisons}

To correct for multiple statistical comparisons, the probability values from the statistical tests were adjusted by false discovery rate. After this adjustment, statistically significant associations remained for the following parameters:

1. Lower spontaneous secretions of all of the studied cytokines (IL-6, IL-10, IL-13, IL-17, IFN- $\gamma$, and TNF- $\alpha$ ) in T1D children around the time of diagnosis in comparison to the healthy children $(P<0.0001, P=0.004$, $P<0.0001, P=0.0031, P<0.0001$, and $P=0.0103$, respectively).

Table 1. Clinical factors that influence the immune response at diagnosis of type 1 diabetes

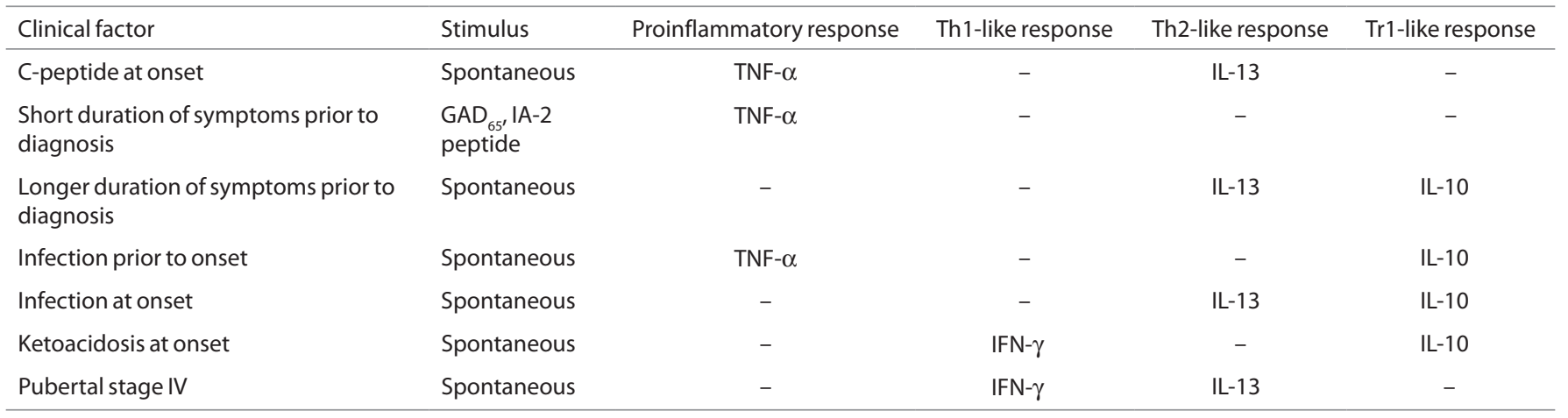

$\mathrm{GAD}_{65^{\prime}}$ glutamic acid decarboxylase; IFN, interferon; IL, interleukin; Th, Thelper; Tr, regulatory $T$ cell; $T N F$, tumor necrosis factor. 
2. Mitogen-induced secretion of IL-10 and IL-17 were generally lower in T1D patients around the time of diagnosis $(P=0.008$ and $P=0.017$, respectively).

3 . Lower secretion of TNF- $\alpha$ was connected to higher dosage of insulin per kilogram body weight, during the first $24 \mathrm{~h}$ following diagnosis $(P=0.01)$.

4. Higher $\mathrm{GAD}_{65}$ protein-specific TNF- $\alpha$ secretion was observed in children with a short duration of symptoms as compared to those with $6 \mathrm{wk}$ and longer duration of symptoms $(P=0.0125)$.

5. PHA-induced TNF- $\alpha$ secretions were higher in patients whose duration of symptoms was about $4 \mathrm{wk}(P=$ 0.0125).

6. Spontaneous secretion of IL-10 and IL-13 was higher in T1D diagnosis preceded by symptom durations of $12-16$ wk $(P=0.014$ and $P=0.007$, respectively).

7. Mitogen-induced IL-13 secretion was higher in those individuals whose T1D diagnosis was preceded by 2 or 4 wk duration of symptoms $(P=0.025$ and $P=0.0125$, respectively).

8. Higher secretion of TNF- $\alpha$ in T1D cases with an infection of $2 \mathrm{wk}$ before the diagnosis $(P=0.0125)$.

9. Higher mitogen-induced secretion of IFN- $\gamma$ and TNF- $\alpha$ in patients diagnosed during the later stages of puberty (IV and V) $(P=0.004$ and $P=0.002$, respectively).

\section{Secretion of IL-17}

Very few subjects had measurable concentrations of spontaneously secreted IL-17 to allow for reliable calculations within the T1D cohort, and as for autoantigen-induced secretions, there were no detectable differences (data not shown).

\section{DISCUSSION}

T1D is a serious diagnosis with the prospect of grave short- and long-term complications and even death if poorly managed. With the gathered clinical and immunological information, we have analyzed whether the metabolic state influences the immune balance. The purpose of this study was to investigate the possible associations and interactions between clinical and immunological parameters that might influence each other close to the diagnosis of T1D. The results were adjusted for multiple statistical comparisons, as it is usually done when testing hypotheses. Since this is more of a hypothesis-generating study, this might be unnecessary. We therefore present both the adjusted and the unadjusted probability values.

In general, T1D children, around the time of diagnosis, showed a low spontaneous secretion of all the studied cytokines (IL-6, IL-10, IL-13, IL-17, IFN- $\gamma$, and TNF- $\alpha$ ) in comparison to healthy children. This is in accordance with the decreased Th1 immunology at T1D onset, which we and others have reported previously $(14,15,18,19)$. It could be speculated that the low, seemingly suppressed, spontaneous cytokine secretions could be an effect of exhaustion of the immune system following the strong immune activation preceding the onset and diagnosis of T1D. A popular idea, connected to the hypothesis that most $\beta$ cells are destroyed at the time of diagnosis, is that the autoreactive immune cells have nothing left to fight and therefore generate less cytokines. In line with this hypotheses, it was observed that children still able to secrete C-peptide at diagnosis showed an immune response including proinflammatory (TNF- $\alpha$ ), Th2-like (IL-13), and, to some extent, also Tr1-like (IL-10) immune markers. This immune response may mirror a still ongoing autoimmune attack of the remaining $\beta$ cells and confirms our previous observation of an inflammatory immune response at the onset of disease (20,21). Moreover, a higher stimulated cytokine secretion seen in T1D, despite low spontaneous secretion, could speculatively be due to the generation of memory cells recognizing the putative autoantigens and thereby augment the immune response upon restimulation in vitro.

It has been shown that an initial low level of $\mathrm{C}$-peptide is a strong risk factor for a decrease in $\beta$-cell function (22). It can be speculated that a more rapid autoimmune progression toward the $\beta$ cells will cause a burst of exhibited autoantigens that maintains the autoimmune process. In line with this argument, it was observed that children with a short duration of symptoms prior to onset had a proinflammatory response by secretion of TNF- $\alpha$ in response to the diabetes-associated autoantigens $\mathrm{GAD}_{65}$ and a peptide of IA-2. Speculatively, these children may have a more rapid and aggressive autoimmune process of the remaining $\beta$ cells. In contrast, children with a prolonged duration of symptoms prior to diagnosis showed a Th2-like (IL-13) and a $\operatorname{Tr} 1$ like (IL-10) immune activity that may implicate a less aggressive autoimmune attack. We have previously shown that still-healthy individuals with a high risk of developing the disease have a Th2-prone immune profile in response to diabetes-associated autoantigens $(14,18)$. This $\operatorname{Th} 2 / \operatorname{Tr} 1$-like immune response may favor survival of $\beta$ cells and thus delays the onset of disease.

It has for long been discussed that enteroviral infections increase in frequency in newly diagnosed T1D patients. Enteroviral infections have been found almost twice as frequent in siblings who developed clinical T1D than in siblings who remained nondiabetic (23). Indication of a recent infection by immunoglobulin $\mathrm{M}$ class enterovirus antibodies have been found to be significantly elevated in young children with newly diagnosed T1D (23). Twenty of the 69 T1D children in this study had had an infection up to a month prior to, or still at, diagnosis. As mentioned above, the most common type of infection reported in this cohort was the common cold accompanied by fever, influenza, and, in a few cases, otitis or gingivitis. One child had a urinary tract infection caused by Escherichia coli. In the present study, increased proinflammatory (TNF- $\alpha$ ) and Tr1-like (IL-10) immune responses were observed in T1D children with an infection prior to onset of the disease. It was also observed that the shorter the interval between preceding infection and T1D diagnosis, the lower the release of $\mathrm{C}$-peptide. It has recently been shown that monocytes represent an additional cellular target for C-peptide anti-inflammatory activity (24). Monocytes are pivotal cells in inflammatory responses as they serve as the principal reservoir of proinflammatory cytokines, e.g., TNF- $\alpha$. These cells are the first to be engaged in nonspecific immune responses such as those triggered by environmental factors, e.g., by infections. 
Thus, monocytes can be one of the sources of the release of the proinflammatory cytokine TNF- $\alpha$ in children with an infection preceding the onset of T1D.

However, children and adolescent with an ongoing infection at the time of diagnosis showed immune responses focused to Th2-like and Tr1-like immune profiles without increased secretion of TNF- $\alpha$. This observation is confirmed by a previous study, showing an increased frequency of enteroviral infections in newly diagnosed T1D patients but with lower frequencies of TNF- $\alpha$ in serum as compared to healthy controls (25).

Children/adolescents diagnosed with T1D during pubertal stage IV were found to have a higher ratio of IL-13/IFN- $\gamma$ as compared to children diagnosed during pubertal stage I. We can only speculate that patients diagnosed with T1D during the later pubertal stages have slower autoimmune processes and less aggressive immune responses. In contrast, youngsters during the later stages of puberty have the ability to respond in a Th1- and proinflammatory-associated manner from in vitro mitogen stimulation that may indicate a more developed immune system.

Studies on how ketoacidosis affect the immune response at diagnosis of T1D are limited. However, it was recently shown that in children with moderate or severe diabetic ketoacidosis at diagnosis, secretion of IL-6 and IL-10 was elevated (26-28). High-sensitive C-reactive protein was also shown to be correlated to the increase in IL-6 secretion indicating an ongoing inflammation (27). This observation is in line with the present finding, showing a higher ratio of spontaneously secreted IL-10/ IFN- $\gamma$ in children suffering from ketoacidosis at diagnosis. It has been speculated that these changes may be responsible for increased capillary perturbation, which could subsequently lead to the development of acute ketoacidosis complications (27).

In this study, all the in vitro analysis have been performed postcryopreservation to exclude any possible differences between freshly and cryopreserved handled cells and also in order to avoid any risk of fluctuation, in time, in the methodology analyzing cytokine secretion. In order to prove that the presented data from cryopreserved PBMCs are trustworthy, we performed the same in vitro culture protocol on a limited subgroup of freshly handled samples. Even if the number of T1D children and healthy controls were very limited, we could still observe lower spontaneous secretions of IL-6, IL-13, and TNF- $\alpha$ in T1D children as compared to healthy controls. This result clearly proves that cryopreserved PBMCs can be used for in vitro culture and that we have been able, in a limited number of samples, to validate our results.

In summary, this study confirms that there is a generally low immune response at diagnosis of T1D. However, high C-peptide, short duration of symptoms, or an infection prior to diagnosis were associated with an increased immune activity, including proinflammatory, Th2-associated, and Tr1associated cytokines. In contrast, ketoacidosis and later pubertal stage at onset of disease were more related to a Th1-prone response. These data describe a general immune dampening at diagnosis of T1D, which seems to be related to the metabolic state close to diagnosis.

\section{METHODS}

\section{Study Population and Collection of Clinical Data}

Sixty-nine newly diagnosed T1D children were included in the study ( 40 females and 29 males; aged $2.3-17 \mathrm{y}$; mean age: $10.6 \mathrm{y}$ ), for the investigation of clinical as well as immunological (cytokines) parameters. As a reference group for the cytokine measurements, blood samples from 30 healthy children (11 females and 19 males; aged 7-15 y; mean age: $11.0 \mathrm{y}$ ) were obtained. None of the healthy children or their first-degree relatives had T1D or any other autoimmune disease, and none had increased levels of GAD 65 or IA-2 autoantibodies.

Sodium-heparinized venous blood samples were obtained from children at diagnosis of T1D or during the following days, and from healthy children at school, possibly during the morning hours to avoid time-of-day differences.

PBMCs were isolated using Ficoll Paque density gradient centrifugation (Pharmacia Biotech, Sollentuna, Sweden) and were cryopreserved in liquid nitrogen until further use, as to the fact that blood samples were taken on different occasions.

Information of clinical status of the patients such as BMI, weight loss, pubertal stage, duration of symptoms, previous and/or ongoing infections, insulin requirement, and ketoacidosis were gathered routinely by the treating clinician. Most of the infections reported were specified as common cold or influenza, as well as a few cases of otitis or gingivitis. C-peptide and $\mathrm{HbAlc}$ were analyzed in sera.

PBMCs, enriched out of sodium-heparinized venous blood samples, collected from a small subgroup including five T1D children (one female and four males; aged 6-14 y; mean age: $10.8 \mathrm{y}$ ) and five healthy children (three females and two males; aged 10-13 y; mean age: $11.4 \mathrm{y}$ ) were in vitro cultured immediately after separation, according to the exact same protocol as for cryopreserved PBMCs.

\section{C-peptide Analysis}

A time-resolved flouroimmunoassay (AutoDELFIA C-peptide kit; PerkinElmer, Wallac, Finland) was used to measure C-peptide levels in serum samples of fasting T1D children. Validation of assays was performed by including a C-peptide control module containing a high-level control as well as medium-level and low-level controls (Immulite; Siemens Healthcare Diagnostics Products, Gwynedd, UK). Automatic measurement and calculation were carried out using the data management software program 1224 MultiCalc (PerkinElmer), and results were expressed in nanomoles per liter with a detection limit of $0.03 \mathrm{nmol} / \mathrm{l}$.

\section{In Vitro Cell Culturing}

Aliquots of one million PBMCs ( 91\% viability for each population) were diluted in 1,000 $\mu \mathrm{l}$ AIM V research-grade serum-free medium (Gibco; Invitrogen, Stockholm, Sweden) supplemented with $2 \mathrm{mmol} / \mathrm{l}$ L-glutamine, $50 \mu \mathrm{g} / \mathrm{l}$ streptomycin sulfate, $10 \mu \mathrm{g} / \mathrm{l}$ gentamicin sulfate, and $2 \times 10^{-5} \mathrm{~mol} / \mathrm{l}$ 2-mercaptoethanol (Sigma, Stockholm, Sweden) and incubated in medium alone (spontaneous secretion) or stimulated with $5 \mu \mathrm{g} / \mathrm{ml}$ of the autoantigens $\mathrm{GAD}_{65}$ (protein; Diamyd Diagnostics AB, Stockholm, Sweden), the synthetic peptide of $\mathrm{GAD}_{65} \mathrm{AA}$ 247-279 (NMYAMMIARFKMFPEVKEKGMAALPRLIAFTSE-OH) with molecular weight 3823.7 (Department of Medical and Physiological Chemistry, University of Uppsala, Sweden), a peptide of heat-shock protein 60 AA 437-460 (DiaPep277, a gift from B. Roep, Leiden University Medical Center, Leiden, The Netherlands) and the mitogen PHA (Sigma), $0.5 \mu \mathrm{g} / \mathrm{ml}$ insulin peptide (AA 9-23, Sigma, Genosys, Canada), and $10 \mu \mathrm{g} / \mathrm{ml} \mathrm{IA-2-peptide} \mathrm{(AA} \mathrm{853-872,} \mathrm{Proimmune,}$ Oxford, UK) (order of priority in case of inadequate cell count; spontaneous, $\mathrm{GAD}_{65}$-protein, IA-2-peptide, heat-shock protein 60 -peptide, insulin-peptide, $\mathrm{GAD}_{65}$-peptide, and finally $\mathrm{PHA}$ ) at $37^{\circ} \mathrm{C}$ in a humidified atmosphere with $5 \% \mathrm{CO}_{2}(14,19,20)$. Cell culture supernatants were harvested after $72 \mathrm{~h}$ of in vitro culture for the analysis of cytokines.

\section{Detection of Cytokines by Multiplex Fluorochrome Technique} (Luminex)

Cytokines (IL-6, IL-10, IL-13, IL-17, IFN- $\gamma$, and TNF- $\alpha$ ) were detected in the cell culture supernatants by multiplex fluorochrome technique (Luminex, Austin, TX) using Bio-Plex Human Cytokine Panel (BioRad Laboratories, Hercules, CA), according to the manufacturer's 
instructions. In all assays, the Bio-Plex Cytokine Reagent Kit (Bio-Rad Laboratories) was used.

Identification and quantification of each bead subset were done using a Luminex ${ }^{100}$ instrument (xMAPtechnology; Luminex). A minimum of 100 beads per region was analyzed. Raw data were analyzed using Starstation Software version 2.3 (Applied Cytometry Systems, Sheffield, UK), and a curve fit was applied to each standard curve according to the manufacturer's instruction. The cutoff for minimum detectable concentrations for each immunological marker was as follows: IL-6: $0.37 \mathrm{pg} / \mathrm{ml}$; IL-10: $0.19 \mathrm{pg} / \mathrm{ml}$; IL-13: $0.36 \mathrm{pg} / \mathrm{ml}$; IL-17: $0.48 \mathrm{pg} / \mathrm{ml} ;$ IFN- $\gamma: 0.40 \mathrm{pg} / \mathrm{ml}$; and TNF- $\alpha: 1.41 \mathrm{pg} / \mathrm{ml}$.

Randomly chosen samples from both the study groups were run in each assay to avoid significance between the groups due to any interassay differences. Specific secretion was obtained by subtraction of the spontaneous secretions from the stimulated secretion. In this study, possible associations between cytokines (single or ratio) and clinical parameters that might influence each other close to the diagnosis of T1D were investigated.

\section{Statistics}

To compare groups, the Mann-Whitney $U$-test and the KruskalWallis tests were used. The analyses on the clinical and immunological parameters were done with linear regression analysis or quintile regression, depending on whether the parameter was normally distributed or not. To correct for multiple statistical comparisons, the probability values from the statistical tests were adjusted by false discovery rate (29). A probability level of $<0.05$ was considered statistically significant. The statistical package Stata 12.0 (Stata, College Station, TX) for PC was used for the statistical analyses.

\section{Ethics}

This study was approved by the Research Ethics Committee of the Faculty of Health Sciences, Linköping University, Sweden. Informed consent was obtained from all study subjects and the parents or the responsible guardians of the children.

\section{ACKNOWLEDGMENTS}

The authors thank Lena Berglert for C-peptide analysis; Hanna Skärstrand and Linnea Jonsson for contributing to the cytokine analysis; research nurses Ann-Marie Sandström and Eva Isaksson; and technician Ingela Johansson for help in the archives.

\section{STATEMENT OF FINANCIAL SUPPORT}

This study was supported by the Swedish Child Diabetes Foundation (Barndiabetesfonden).

Disclosure: There are no conflicts of interest in the presented study, neither regarding the collection, analysis and interpretation of data, nor the writing of the report and the decision to submit for publication. Anna Rydén is an external, postdoctoral scientist at Novo Nordisk, Seattle, WA.

\section{REFERENCES}

1. Nordwall M, Ludvigsson J. Clinical manifestations and beta cell function in Swedish diabetic children have remained unchanged during the last 25 years. Diabetes Metab Res Rev 2008;24:472-9.

2. American Diabetes Association. Diagnosis and classification of diabetes mellitus. Diabetes Care 2008;31:Suppl 1:S55-60.

3. Steffes MW, Sibley S, Jackson M, Thomas W. Beta-cell function and the development of diabetes-related complications in the diabetes control and complications trial. Diabetes Care 2003;26:832-6.

4. Scalia R, Coyle KM, Levine BJ, Booth G, Lefer AM. C-peptide inhibits leukocyte-endothelium interaction in the microcirculation during acute endothelial dysfunction. FASEB J 2000;14:2357-64.

5. Leslie RD, Atkinson MA, Notkins AL. Autoantigens IA-2 and GAD in Type I (insulin-dependent) diabetes. Diabetologia 1999;42:3-14.

6. Elias D, Prigozin H, Polak N, Rapoport M, Lohse AW, Cohen IR. Autoimmune diabetes induced by the beta-cell toxin STZ. Immunity to the $60-\mathrm{kDa}$ heat shock protein and to insulin. Diabetes $1994 ; 43$ : 992-8.
7. Wenzlau JM, Juhl K, Yu L, et al. The cation efflux transporter ZnT8 (Slc30A8) is a major autoantigen in human type 1 diabetes. Proc Natl Acad Sci USA 2007;104:17040-5.

8. Christen U, von Herrath MG. Manipulating the type 1 vs type 2 balance in type 1 diabetes. Immunol Res 2004;30:309-25.

9. Eisenbarth GS. Type I diabetes mellitus. A chronic autoimmune disease. N Engl J Med 1986;314:1360-8.

10. Castaño L, Eisenbarth GS. Type-I diabetes: a chronic autoimmune disease of human, mouse, and rat. Annu Rev Immunol 1990;8:647-79.

11. Bradley LM, Asensio VC, Schioetz LK, et al. Islet-specific Th1, but not Th2, cells secrete multiple chemokines and promote rapid induction of autoimmune diabetes. J Immunol 1999;162:2511-20.

12. Honkanen J, Nieminen JK, Gao R, et al. IL-17 immunity in human type 1 diabetes. J Immunol 2010;185:1959-67.

13. Berman MA, Sandborg CI, Wang Z, et al. Decreased IL-4 production in new onset type I insulin-dependent diabetes mellitus. J Immunol 1996;157:4690-6.

14. Karlsson MG, Lawesson SS, Ludvigsson J. Th1-like dominance in high-risk first-degree relatives of type I diabetic patients. Diabetologia 2000;43:7429.

15. Halminen M, Simell O, Knip M, Ilonen J. Cytokine expression in unstimulated PBMC of children with type 1 diabetes and subjects positive for diabetes-associated autoantibodies. Scand J Immunol 2001;53:510-3.

16. Taylor A, Verhagen J, Blaser K, Akdis M, Akdis CA. Mechanisms of immune suppression by interleukin-10 and transforming growth factorbeta: the role of T regulatory cells. Immunology 2006;117:433-42.

17. Ludvigsson J, Heding LG. C-peptide in children with juvenile diabetes. A preliminary report. Diabetologia 1976;12:627-30.

18. Hedman M, Ludvigsson J, Faresjö MK. Nicotinamide reduces high secretion of IFN-gamma in high-risk relatives even though it does not prevent type 1 diabetes. J Interferon Cytokine Res 2006;26:207-13.

19. Karlsson Faresjö MG, Ernerudh J, Ludvigsson J. Cytokine profile in children during the first 3 months after the diagnosis of type 1 diabetes. Scand J Immunol 2004;59:517-26.

20. Stechova K, Bohmova K, Vrabelova Z, et al. High T-helper-1 cytokines but low T-helper-3 cytokines, inflammatory cytokines and chemokines in children with high risk of developing type 1 diabetes. Diabetes Metab Res Rev 2007;23:462-71.

21. Ryden A, Stechova K, Durilova M, Faresjö M. Switch from a dominant Th1-associated immune profile during the pre-diabetic phase in favour of a temporary increase of a Th3-associated and inflammatory immune profile at the onset of type 1 diabetes. Diabetes Metab Res Rev 2009;25:335-43.

22. Törn C, Landin-Olsson M, Lernmark A, et al. Prognostic factors for the course of beta cell function in autoimmune diabetes. J Clin Endocrinol Metab 2000;85:4619-23.

23. Hyöty $H$, Hiltunen $M$, Knip $M$, et al. A prospective study of the role of coxsackie $\mathrm{B}$ and other enterovirus infections in the pathogenesis of IDDM. Childhood Diabetes in Finland (DiMe) Study Group. Diabetes 1995;44:652-7.

24. Haidet J, Cifarelli V, Trucco M, Luppi P. C-peptide reduces pro-inflammatory cytokine secretion in LPS-stimulated U937 monocytes in condition of hyperglycemia. Inflamm Res 2012;61:27-35.

25. Elfaitouri A, Berg AK, Frisk G, Yin H, Tuvemo T, Blomberg J. Recent enterovirus infection in type 1 diabetes: evidence with a novel IgM method. J Med Virol 2007;79:1861-7.

26. Hoffman WH, Burek CL, Waller JL, Fisher LE, Khichi M, Mellick LB. Cytokine response to diabetic ketoacidosis and its treatment. Clin Immunol 2003;108:175-81.

27. Karavanaki K, Karanika E, Georga S, et al. Cytokine response to diabetic ketoacidosis (DKA) in children with type 1 diabetes (T1DM). Endocr J 2011;58:1045-53.

28. Karavanaki K, Kakleas K, Georga S, et al. Plasma high sensitivity C-reactive protein and its relationship with cytokine levels in children with newly diagnosed type 1 diabetes and ketoacidosis. Clin Biochem 2012;45:1383-8.

29. Curran-Everett D. Multiple comparisons: philosophies and illustrations. Am J Physiol Regul Integr Comp Physiol 2000;279:R1-8. 